\title{
Insertable Surgical Imaging Device with Pan, Tilt, Zoom, and Lighting
}

\author{
Tie Hu, Peter K. Allen, Nancy J. Hogle and Dennis L. Fowler
}

\begin{abstract}
This paper describes work we have done in developing an insertable surgical imaging device with multiple degrees-of-freedom for minimally invasive surgery. The device is fully insertable into the abdomen using standard $12 \mathrm{~mm}$ trocars. It consists of a modular camera and lens system which has pan and tilt capability provided by 2 small DC servo motors. It also has its own integrated lighting system that is part of the camera assembly. Once the camera is inserted into the abdomen, the insertion port is available for additional tooling, motivating the idea of single port surgery. A third zoom axis has been designed for the camera as well, allowing close-up and far-away imaging of surgical sites with a single camera unit.
\end{abstract}

In animal tests with the device we have performed surgical procedures including cholecystectomy, appendectomy, running (measuring) the bowel, suturing, and nephrectomy. The tests show that the new device is:

- Easier and more intuitive to use than a standard laparoscope.

- Joystick operation requires no specialized operator training.

- Field of view and access to relevant regions of the body were superior to a standard laparoscope using a single port.

- Time to perform procedures was better or equivalent to a standard laparoscope.

We believe these insertable platforms will be an integral part of future surgical systems. The platforms can be used with tooling as well as imaging systems, allowing many surgical procedures to be done using such a platform.

\section{INTRODUCTION}

Minimally Invasive Surgery (MIS) encompasses laparoscopy, thoracoscopy, arthroscopy, intraluminal endoscopy, endovascular techniques, catheter-based cardiac techniques, and interventional radiology[2], and has grown rapidly over the last two decades. In 1992, $70 \%$ of all cholecystectomies (gall bladder removal) in the United States, Europe, and Japan were performed using laparoscopic techniques[1]. In laparoscopic surgery, the surgeon first cuts several small incisions in the abdomen, and inserts trocars (small tubes) through the incisions. Carbon dioxide gas is pumped into the abdomen to create a larger volume of space for the operation and visualization. By viewing the image from the laparoscope which is inserted into the body through the trocar, the surgeon operates the laparoscopic tools to perform surgery. Laparoscopic surgery has many

This work was supported by NIH grant 1R21EB004999-01A1

Tie Hu is with Department of Computer Science, Columbia University, New York, NY10027 USA tie@cs. columbia.edu

Peter K. Allen is with Department of Computer Science, Columbia University New York, NY 10027 USA allen@ cs. columbia.edu

Nancy J. Hogle is with Department of Surgery, Columbia University New York, NY 10032 USA nh2106@columbia.edu

Dennis L. Fowler is with Department of Surgery, Columbia University New York, NY 10032 USA dlf91@columbia.edu benefits, such as small incisions, less pain and trauma to the patients, faster recovery time, and lower health care cost. However, this technique drastically increases the complexity of a surgeons' task because of the rigid, sticklike instruments, impaired depth perception, loss of sense of touch (haptics) and the difficulty in varying the perspective view of the operative field[1].

Robotic surgery is considered as the future of surgery[13]. Robots for MIS could greatly increase the dexterity and fine motion capabilities of a surgeon during an operation, decrease the tremor of a surgeon's hand, and enable remote operation[12], [18], [11], [9], [7]. Robotic surgery still comprises only a very small portion of all minimally invasive surgery. Current surgical robots tend to be extremely expensive with the price of a da Vinci robot (Intuitive Surgical) being typically over a million dollars. In addition, the size of many current surgical robots is extremely large, tending to occupy a large portion of the sterile field of an operating room.

There is a definite need to develop a surgical robot which is more compact and less expensive than existing systems. Our goal is to enhance and improve surgical procedures by placing small, mobile, multi-function platforms inside the body that can begin to assume some of the tasks associated with surgery. We want to create a feedback loop between new, insertable sensor technology and effectors we are developing, with both surgeons and computers in the informationprocessing/control loop. We envision surgery in the future as radically different from today. This is clearly a trend that has been well-established as minimal-access surgical procedures continue to expand. Accompanying this expansion have been new thrusts in computer and robotic technologies that make automated surgery, if not feasible, an approachable goal. It is not difficult to foresee teams of insertable robots performing surgical tasks inside the body under both surgeon and computer control. The benefits of such an approach are well documented: greater precision, less trauma to the patient, and improved outcomes. One factor limiting this expansion is that the laparoscopic paradigm of pushing long sticks into small openings is still the state-of-the-art, even among surgical robots such as DaVinci. While this paradigm has been enormously successful, and has spurred development of new methods and devices, it is ultimately limiting in what it can achieve. Our intent is to go beyond this paradigm, and remotize sensors and effectors into the body cavity where they can perform surgical and imaging tasks unfettered by traditional endoscopic instrument design.

The basic architecture of the endoscope has not been fundamentally changed since the invention of the rod-lens 
by Hopkins and cold light source of fiber optics by Karl Storz in 1950's[16]. Traditional endoscope uses the fiberoptics to deliver the light into the abdomen and the rodlens to transmit the image back to the CCD camera sensor. This approach has a number of limitations, such as narrow imaging, limited work space, counter intuitive motion and additional incisions for the endoscope. Since the surgeon is generally working with both hands holding other instruments, an assistant is necessary to hold the endoscope steady and move it as required. Recent work in robotics has sought to automate that task. One commercially available system called AESOP can orient a traditional endoscope using a robotic arm that is controlled by spoken commands[17]. While this takes the burden off the assistant and provides a much more stable image, it still occupies a large part of the operating room floor. The similar principle is used in da Vinci surgical robots[18]. A simpler robotic endoscope manipulator that can be placed directly over the insertion point was developed at INRIA[19]. However, none of these systems addresses the fundamentally limited range of motion of the endoscope. The fulcrum point created by the abdominal wall restricts the motion of the scope to 4 degrees of freedom, so that the only translation possible is along the camera axis.

There is some related research on new designs for endoscopes. One system uses a traditional rigid rod endoscope but adds a motor that rotates a 90-degree mirror at the end of the scope to provide an additional degree of freedom [20]. Another system is essentially a multi-link arm that positions a camera using piezoelectric actuators [21]. Theoretically this robot would provide many different viewing angles for an attached camera, but the authors provide no information about the safety of using piezoelectoric electric elements, and do not appear to have attempted any tests within living animals or humans. The pill camera [22] is an example of a camera that operates entirely within the body. It is able to image sections of the small intestine that an endoscope cannot reach. However, it does not have any means of actuation and simply relies on peristalsis for locomotion. Magnetic anchoring was used to maneuver the locomotion of a micro camera in the body [23]. Since there are no additional actuators in the camera, the view point is limited by the camera orientation.

Other examples of new ideas in designing surgical robots include Dachs and Peine [4] who developed a 6 DOF surgical robot which eliminates the dependence on pivoting about the incision point. Sastry et.al. [5] presented a milli-robot for remote, minimally invasive surgery.

We have been focusing on developing an inexpensive, insertable endoscopic camera with multiple degrees-offreedoms (DOFs). In this paper, we describe our insertable Pan/Tilt endoscope with integrated light source that we have built and and tested in five in vivo animal tests. Surgeons have used this device to perform laparoscopic appendectomy, cholecystectomy, running (measuring) the bowel, suturing, and nephrectomy. The results show that the device is easier to use and control than a standard laparoscope. Our imaging device only requires a single access port and has more flexibility, as it is inside the body cavity and can obtain images from a number of controllable directions. There is no need for extensive training with this device as with a standard laparoscope since it is operated by a simple joystick. Standard laparoscopes have counter-intuitive motions due to the pivoting about the insertion point (e.g. to move the laparoscope to the right, the external part of the unit is moved to the left, pivoting on the insertion point). This can cause confusion for untrained operators. Our device can image a larger field of view than traditional laparoscopes, allowing the surgeon greater flexibility in seeing the inside of the abdominal cavity. Our tests have also shown that zooming capabilities are desirable for such a device, and we also present a design for a zooming capability that will add an extra DOF to our device, extending its utility during surgery.

\section{Prototype Device}

\section{A. New Prototype Imaging Device}

Our initial work [24] in designing such an imaging system created a device with 2 cameras and 5-DOF (independent pan and translation axes for each of two cameras plus a common tilt axis). A single camera, 3-DOF version was successfully tested with surgical fellows in a laparoscopic trainer mockup. These quantitative tests using the MISTELS (McGill Inanimate System for the Training and Evaluation of Laparoscopic Skill) tasks [28] showed the device was able to carry out typical minimally invasive surgical tasks equivalent to using a standard laparoscope, with no loss of function[25]. Based upon this design, we have designed a second generation device that improves upon the design of our initial device described above. Our design goals for the new prototype included reducing the device size (from $22 \mathrm{~mm}$ to $11 \mathrm{~mm}$ in diameter) and the inclusion of an integrated light source. To reduce the device size to allow it to be inserted through a $12 \mathrm{~mm}$ trocar, we removed 1 camera and the translation axis. We have also added an LED light source to the device[14]. The total length of the device is about $110 \mathrm{~mm}$, and the diameter is about $11 \mathrm{~mm}$ and can be inserted into a standard $12 \mathrm{~mm}$ trocar.

We make use of modular design to make the device components interchangeable and extendable. The current system includes a user-friendly interface, making it easier to control the camera's DOF using natural motions. It consists of a Pan/Tilt motorized CCD camera with illumination components, control interface driver, PC, and Joystick controller. After the surgeon anchors the camera onto the abdomen wall, he can use the Joystick to position the camera to the desired surgical viewpoint using the Pan and Tilt motions. The intensity of illumination can be adjusted manually through the control panel. Figure 1 shows images of the implemented prototype device, with integrated lighting and pan/tilt axes.

Figure 2. shows the CAD model of device. In the side view, the shaft of the tilt motor(smoovy brushless DC motor $(0513 \mathrm{G})$ with $625: 1$ planetary gearhead(Series 06A)) is coupled to the external stainless shell, which is used as the mounting base of the device on the abdominal wall. The pan motor is the same as the tilt motor, and is coupled to 


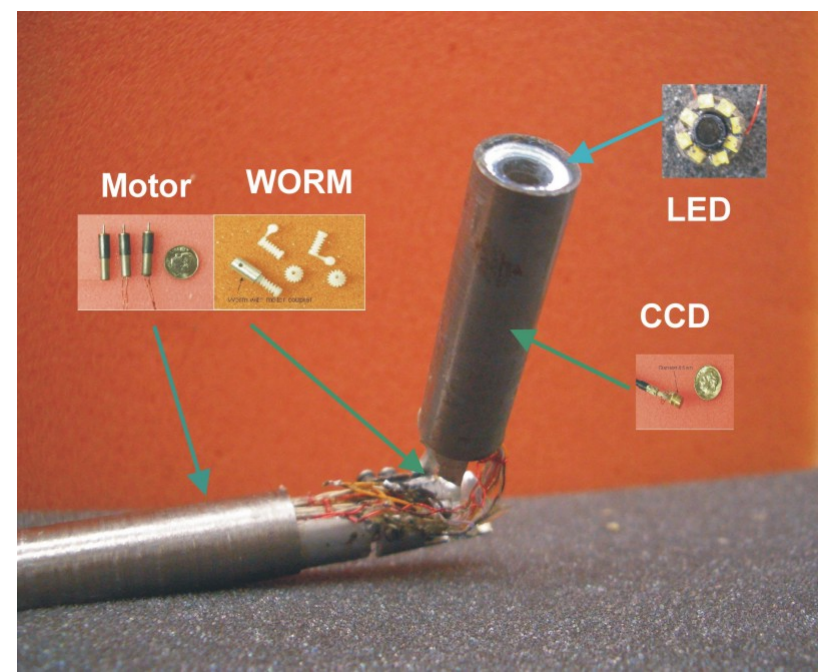

Fig. 1. Implemented Prototype device with LED lighting and pan/tilt axes.

a worm gear. This gear (KLEISS Gear, Inc) has a reduction ratio of 16:1. The worm gear mechanism can transverse the motion in a compact space and increase the output torque. Our design provides a panning range of $120 \circ$ and a tilt range of over 90 .

The camera module contains a lens, CCD sensor and LED light source. We use a miniature pin-hole lens (PTS 5.0 from Universe Kogaku America) with appropriate optical parameters for our device. This camera uses a $\frac{1}{4}$ in. CCD chip and has a very small package size. We selected Luxeon Portable PWT white LED (LXCL_PWT1) as the illumination unit of the device. The LED light source we have designed and constructed consists of a custom made printed circuit board with 8 LEDs. It has a size of $9 \mathrm{~mm}$ in external diameter, $5 \mathrm{~mm}$ in internal diameter, and $3 \mathrm{~mm}$ in thickness. The 8 LED's are serially connected and soldered in a circular printed circuit board. It can deliver a total of 208 lumens of light.

\section{B. Zoom Mechanism}

Our initial tests showed that zooming capabilities are highly important for many surgical tasks. Traditional laparoscopes do not have a zoom mechanism, however, the surgeon can adjust the zoom of the image view by moving the laparoscope in and out through the port. We set up a specification for an endoscope camera by measuring a laparoscope (Karl Storz 26003 AA coupled to a Karl Storz telecam 20212130U NTSC). These parameters are an appropriate design goal for the zoom mechanism. The Storz system has a measurable minimum focus distance of $30 \mathrm{~mm}$ and a maximum focus distance of $160 \mathrm{~mm}$. The measured view angle is 53 degree. We also identified that $40 \mathrm{~mm}$ is an optimal viewing distance (the distance between the lens and the object) for fine dissection, and the optimum viewing distance for gross manipulation is $100 \mathrm{~mm}$. Our design for a zoom mechanism is a translation axis which can move the whole camera module forward and backward and is integrated with the current pan/tilt endoscope. In our current

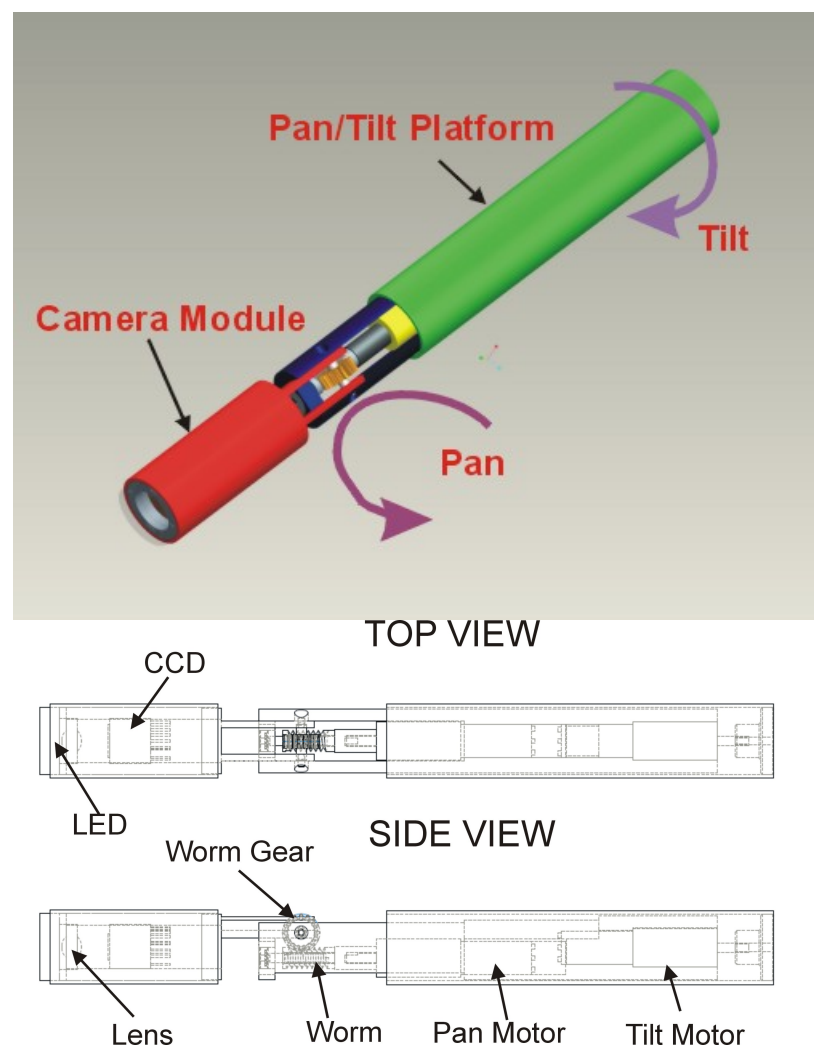

Fig. 2. CAD Model of Implemented Prototype device with LED lighting and pan/tilt axes.

TOP VIEW

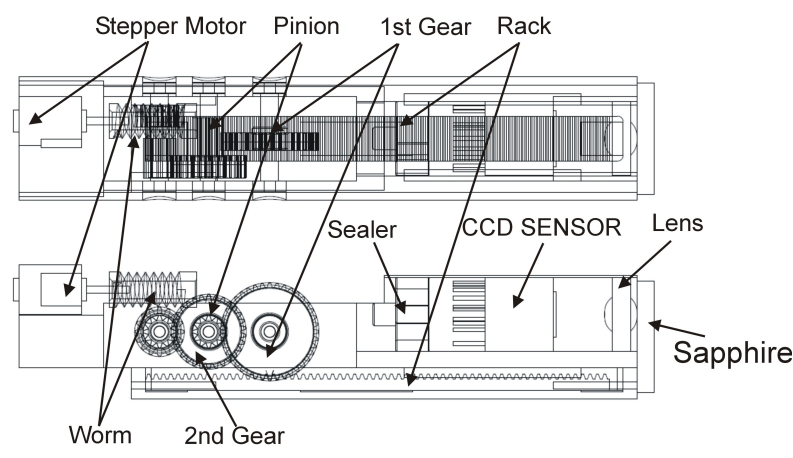

SIDE VIEW

Fig. 3. CAD Model of Zoom Mechanism.

device we use a miniature pin-hole lens with appropriate optical parameters for our device. The focal length of the lens is $5.0 \mathrm{~mm}$ and $\mathrm{F}$ number is 4.0 . We use a $\frac{1}{4}$ in. color video CCD camera head with diameter of $6.5 \mathrm{~mm}$. The camera has active pixels of 752(H) X 582(V) at PAL system, which can provide $450 \mathrm{TV}$ lines in horizontal resolution and 420 TV lines in vertical resolution. The camera is fabricated with the optimum focus distance of $40 \mathrm{~mm}$ according to our determination of optimal viewing distance. Because the optimal viewing distance is variable at different times during an operation, the camera cannot always obtain the best 
image. This has necessitated the development of the zoom capability.

Our zoom mechanism is designed to manipulate the camera forward and backward. A rack and pinion mechanism was chosen as the basic mechanical structure for zooming to achieve a compact size (Side View of Figure 3). A $4.5 \mathrm{~mm}$ miniature stepper motor $(0.08 \mathrm{mNm}$ maximum torque) is used as the actuator to drive the pinion. The zooming distance is $20 \mathrm{~mm}$. The entire zoom package is $12 \mathrm{~mm}$ in diameter and $56 \mathrm{~mm}$ in length. Figure 3 shows the CAD model of the zoom mechanism. It is constructed of a camera module, zoom components and an external shell. To maximize the output torque, 3 sets of gears are used in the design. The 1st gear is a spur gear with 120 Diametral Pitch and 40 teeth. It rotates on a rack, which is mounted on a support which is attached to the external shell. When the motor rotates, the pinion gear travels along the rack, moving the camera module forward and backward along the external shell. A pinion with 120 Diametral Pitch and 12 teeth is matched with 1st gear. 2nd gear(120 Diametral Pitch, 30 teeth) is mounted on the same shaft with this pinion. A pinion with 120 Diametral Pitch and 12 teeth is mounted on the same shaft as the worm. This pinion is matched with 2nd gear. The worm is mounted on the shaft of motor. The ratio of worm gear is 16:1. Finally, we get a total speed reduction of 133:1 with this design, which we are currently testing in animal trials.

\section{EXPERIMENTS AND RESULTS}

We have performed five in vivo porcine animal tests with our device. A laparoscopic surgeon (Fowler) used this device to perform a number of surgical procedures, including cholecystectomy, appendectomy, running (measuring) the bowel, suturing, and nephrectomy (kidney removal). Since this test animal species does not have an appendix as a human, resecting part of the colon was used to simulate an appendectomy. We present results from two of the tests below.

\section{A. Mounting the camera}

Each experiment started with the mounting of the imaging device. A porcine was under general anesthesia. A surgeon first cut small incisions in the abdominal body, then inserted trocars into the incisions. Carbon dioxide gas was pumped into the abdomen to inflate the abdominal cavity. A standard laparoscope was inserted into one trocar, and the image from this laparoscope was used to guide the mounting and orientation of our new imaging device. Figure 4 shows the mounting procedure of our imaging device onto abdominal wall as viewed from the standard laparoscope. The surgeon inserted our device into the body through a trocar. Then a needle with braided silk was inserted through the abdominal skin, which was approximately on top of the imaging device. Next, using a standard laparoscopic gripper, the needle and suture were looped around the tube of the imaging device, and pushed back through the abdominal wall (Figure 4, left.). The braided silk was then tied off on the outside of the abdomen, securing the new device to the interior of the abdominal wall (Figure 4, center). The insertion trocar only contained the power and imaging wires which do not fully occupy the trocar diameter, allowing additional tooling to be used through the same port. Once fixed in place, the device was used to perform the experiments described below.

We have also experimented with other ways to fix the device onto the wall of the abdomen. One method, which we have implemented and used in other animal experiments, is to use an external holding mechanism. This mechanism has a rotational attachment which holds the tilt motor end of the device. When the surgeon grasps the handle of the mechanism, this attachment can rotate 90 degrees. After the device is deployed into the abdomen through the trocar, the surgeon can pull the handle and rotate the device 90 degrees so it is up against the abdominal wall. The disadvantage to this system is that the mechanism fills the trocar space. Another method is to use magnetic anchoring [23]. Two internal magnetic pads can be installed in the ends of device. When the device is fully deployed into the abdomen, the surgeon can use external magnetic components to fix and also maneuver the locomotion of device outside of body. An advantage of this method is it is non-invasive, however, the intensity of the magnetic field will decrease with the increase of the abdomen's thickness, making it not suitable for all patients..

\section{B. Experiment I}

In this experiment, we used the new device and the integrated light source to perform a cholecystectomy and appendectomy. Figure 4 (right) shows our imaging device in the abdomen, exercising the tilt axis for viewing. These images were taken by a standard laparoscope. During the surgery, a person without laparoscopic training was operating the joystick controller by following the commands from surgeon. The surgeon's qualitative assessment of the device was very good, and the cholecystectomy was successfully carried out. Although there was sufficient light to perform the procedures from our integrated lighting we plan to add additional lighting using more powerful LED's to enhance the images.

\section{Experiment II}

In this experiment we performed a number of laparoscopic surgical procedures and compared the timings of each operation with using 1) a standard laparoscope and 2) our new device. One of the authors (Fowler) performed the surgical procedures and personnel without laparoscopic training operated each of the devices. Figure 5 shows a series of images from the new device during an appendectomy. The device was able to pan and tilt easily to accomodate the surgeon's need for new views of the surgical site. Figure 5 also shows the images of running the bowel from our device. During this procedure, the surgeon used a flexible ruler to measure the length of bowel. By following the motion of tools, the device can track the whole procedure. Figure 6 shows the images of a suturing procedure and of a nephrectomy using the imaging 

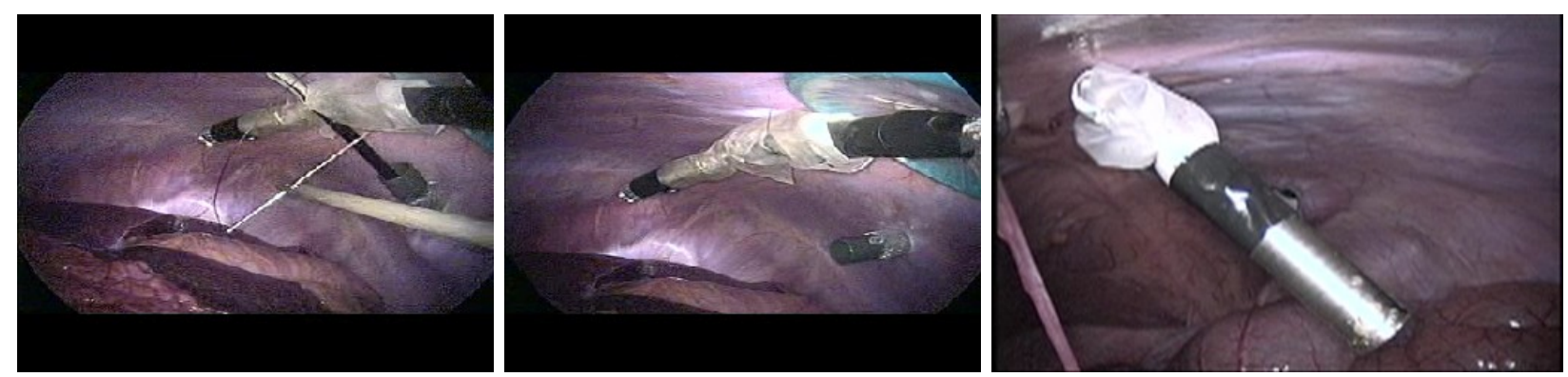

Fig. 4. left: Needle looping around device for attachment. Center: Device firmly attached to abdominal wall. Right: Imaging device in abdominal cavity and tilt axis operating

device. This was a more complicated procedure that required a good deal of camera movement. The pan/tilt feature worked well to provide a range of views of the site as different parts of the procedure were performed. We were only able to perform 1 nephrectomy on the animal, so we do not have a comparison timing for using a standard laparoscope in this procedure.

Table 1 shows the timings of each procedure for both a standard laparoscope and our new device. In all cases, using the new device did not affect the surgeon's ability to perform the procedure efficiently, and in 2 cases, it sped up the procedure. Qualitatively, the imagery was very good, and the ease of control using intuitive commands (move left, right, up, down) with a joystick made operational procedure simple. The experiments suggest that the device is easier to use than a normal laparoscope and that there is no need for special training of the device operator to use the device in clinical procedures. In addition, by using the pan/tilt axes the device can provide a broader/larger field of view than a traditional laparoscope. This suggests that procedures that currently need multiple incisions for multiple laparoscope ports may be able to be performed using a single port and the new insertable camera device.

Table 1: Procedure Timings

\begin{tabular}{|c|c|c|}
\hline Procedure & Device & Time (min) \\
\hline Running Bowel & Laparoscope & $4: 20$ \\
\hline Running Bowel & Robot & $3: 30$ \\
\hline Appendectomy & Laparoscope & $2: 20$ \\
\hline Appendectomy & Robot & $2: 20$ \\
\hline Suturing & Laparoscope & $5: 00$ \\
\hline Suturing & Robot & $4: 00$ \\
\hline Nephrectomy & Robot & $21: 00$ \\
\hline
\end{tabular}

\section{CONCLUSIONS AND FUTURE WORK}

This paper describes a new fully insertable robotic surgical imaging device. The device is part of an effort to create totally insertable surgical imaging systems which do not require a dedicated surgical port, and allow more flexibility and DOF's for viewing. The device has controllable pan/tilt axes, and has been used in-vivo animal experiments which included cholecystectomy, appendectomy, running the bowel, suturing, and nephrectomy. The results suggest that the device is:

- Easier and more intuitive to use than a standard laparoscope.

- Joystick operation requires no specialized operator training.

- Field of view and access to relevant regions of the body superior to a standard laparoscope using a single port.

- Time to perform procedures was better or equivalent to a standard laparoscope.

We believe these insertable platforms will be an integral part of future surgical systems. The platforms can be used with tooling as well as imaging systems, allowing many surgical procedures to be done using such a platform. The system can be extended to a multi-functional surgical robot with detachable end-effectors (grasper, cutting, dissection and scissor). Because the systems are insertable, a single surgical port can be used to introduce multiple imaging and tooling platforms into a patient. In addition, we have built our camera/lens/lighting package in a modular manner, allowing us to design a 2 camera system that can provide stereo 3D views of the site.

One of our design goals is to simplify the operation and control of the imaging system. One possible approach to controlling the cameras would be to use a hybrid controller, which allows the surgeon to control some of the degreesof-freedom (DOF) of the device and an autonomous system, which controls the remaining DOF. For example, the autonomous system can control pan/tilt on the camera to keep a surgeon-identified organ in view, while the surgeon simultaneously may translate the camera to obtain a better viewing angle - all the while keeping the organ centered in the viewing field. We have developed hybrid controllers and mechanisms similar to this for robotic work-cell inspection [27] and believe we can transfer these methods for use with this device.

\section{REFERENCES}

[1] R. H. Taylor, etl, ComputerIntegrated Surgery: Technology and Clinical Applications Cambrisge, MA: The MIT Press; 1996.

[2] Mark Vierra, "Minimally Invasive Surgery,"Annu.Rev.Med.,vol.46, pp147-58,1995.

[3] P.Dario, C. Paggetti, N.Troisfontaine, E. Papa, T.Ciucci, M.C.Carrozza, and M.Marcacci,"A Miniature Steerable End-Effector for application in an integrated system for computer-assisted arthroscopy,"ICRA 97, Albuquerque, New Mexico, 1997. 

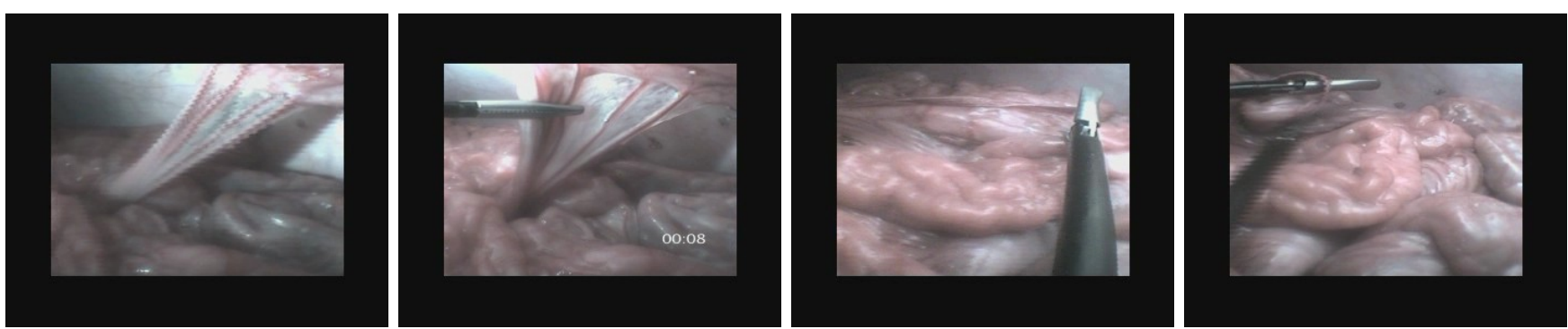

Fig. 5. Images of appendectomy and running the bowel
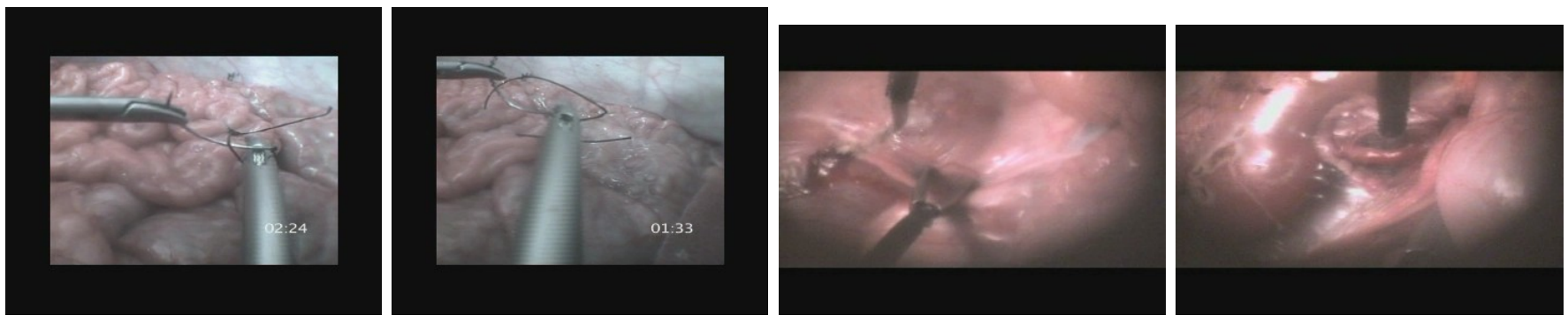

Fig. 6. Images of suturing and nephrectomy

[4] Gregory W. Dachs II and William J.Peine," A Novel Surgical Robot Design: Minimizing the Operating Envelope Within the Sterile Field,"Proceedings of the 28th IEEE EMBS Annual International Conference, New York City, USA, Aug 30-Sept 3, 2006.

[5] S.S.Sastry,M.Cohn, F.Tendick,'Milli-robotics for remote, minimally invasive surgery", Robotics and Autonomous Systems, vol.21, pp.305316,1997.

[6] J.Peirs, D.Reynaerts, H.Van Brussel,"A miniature manipulator for integration in a self-propelling endoscope",Sensors and Actuators, vol.92,pp.343-349, 2001.

[7] D. Oleynikov, M. Rentschler, M. Hadzialic, A. Dumpert, J. Platt, S. Farritor, "Miniature Robots Can Assist in Laparoscopic Cholecystectomy", Journal of Surgical Endoscopy, vol.19, pp. 473-476, 2005.

[8] Daniel B. Jones, and Nathaniel J. Soper, "Complications of Laparoscopic Cholecystectomy", Annu. Rev. Med ,vol.47,pp.31-44,1996.

[9] J.M.Sackier and Y.Wang, "Robotically assisted laparoscopic surgery: From concept to development",Surgical Endoscopy, vol.8,pp.6366,1994 .

[10] J.Rosen, J.D.Brown, L.Chang, M.Barreca, M.Sinanan, and B.Hannaford,'The BlueDRAGON-a system for measuring the kinematics and dynamics of minimally invasive surgical tools in-vivo",ICRA 2002, pp 1876-1881.

[11] R.H.Taylor, J.Funda, B.Eldridge, K.Gruben, D.LaRose, S.Gomory,M.Talamini, L.R.Kavouddi, and J.Anderson,"A telerobotic assistant for laparoscopic surgery", IEEE.Eng.Med.Biol.Mag., vol.14,pp.182-192,1999.

[12] M.Ghodoussi, S.E.Butner, and Y.Wang,"Robotic surgery-the transatlantic case",ICRA 2002 Automation,pp 1882-1888.

[13] Russell H.Taylor, and Dan Stoianovici," Medical Robotics in Computer-Integrated Surgery", IEEE Transactions on Robotics and Automation, Vol.19, No.5

[14] Tie Hu, Peter K. Allen, Dennis L. Fowler, "In-Vivo Pan/Tilt Endoscope with Integrated Light Source", Int. Conf, on Intelligent Robots and Systems (IROS), Oct 29-Nov 2, 2007, San Diego.

[15] Intuitive Investor FAQ ,"http://investor.intuitivesurgical.com".

[16] G. J. Fuchs, "Milestones in endoscope design for minimally invasive urologic surgery: the sentinel role of a pioneer," Surg Endosc, vol. 20, pp. 493-499, 2006.

[17] W. P. Geis, H. C. Kim, E. J. B. Jr., P. C. McAfee, and Y. Wang, "Robotic arm enhancement to accommodate improved efficiency and decreased resource utilization in complex minimally invasive surgical procedures," Proc. Medicine Meets Virtual Reality: Health Care in the Information Age,pp.471-481, 1996.

[18] G. Guthart and K. Salisbury, "The IntuitiveTM Telesurgery Sys- tem: Overview and Application," IEEE International Conference on Robotics and Automation,pp.618-621, 2000.

[19] P. Berkelman, P. Cinquin, J. Troccaz, J. Ayoubi, C. Letoublon, and F. Bouchard, "A compact, compliant laparoscopic endoscope manipulator," ICRA 2002,pp.1870-1875.

[20] L. M. Gao, Y. Chen, L. M. Lin, and G. Z. Yan, "Micro motor based a new type of endoscope," Intl. Conf of the IEEE Engineering in Medicine and Biology Society,vol.20(4),pp.1822-1825, 1998.

[21] K. Ikuta, M. Nokata, and S. Aritomi, "Biomedical micro robots driven by miniature cybernetic actuator," IEEE Workshop on Micro Electromechanical Systems, pp.263-268, 1994.

[22] M. Yu, "M2A? Capsule endoscopy: A breakthrough diagnostic tool for small intestine imaging," Gastroenterology Nursing, vol. 25, pp. pp. 24-27, 2002.

[23] S. Park, R. Bergs, R. Eberhart, L. Baker, R. Fernandez, and J. A. Cadeddu, "Trocar-less Instrumentation for Laparoscopy Magnetic Positioning of Intra-abdominal Camera and Retractor," Annals of Surgery, vol. 245,Issue.3,pp.379-384, 2007.

[24] A. Miller, P. Allen, and D. Fowler, "In-Vivo Stereoscopic Imaging System with 5 Degrees-of-Freedom for Minimal Access Surgery," Medicine Meets Virtual Reality Conference (MMVR), 2004.

[25] V. E. M. Strong, N. J. Hogle, and D. L. Fowler, "Efficacy of Novel Robotic Camera vs a Standard Laparoscopic Camera," Surgical Innovation, vol. 12, 2005.

[26] W. J. Smith, Modern Lens Design, McGraw-Hill, 2004

[27] P. Oh and P.Allen, "Visual Servoing by Partitioning Degrees-ofFreedom," IEEE Trans. on Robotics and Automation, vol. 17, pp.1-17, February 2001

[28] Derossis, A.M., Fried, G.M., Abrahamowicz, M., Sigman, H.H., Barkun, J.S., Meakins, J.L," Development of a model for training and evaluation of laparoscopic skills". American Journal of Surgery 175(6):482-487, 1998. 